\title{
Dessorção do herbicida atrazina e atividade microbiana em duas classes de solos do Estado do Rio Grande do Sul
}

\author{
Dessorption of the herbicide atrazine and microbial activity in two soil classes of Rio Grande do Sul State
}

\author{
Adriana Regina Bohn Kleinschmitt ${ }^{1}$ Deborah Pinheiro Dick ${ }^{2}$ Pedro Alberto Selbach $^{3}$ \\ Marlon dos Santos ${ }^{4}$
}

\section{RESUMO}

O objetivo deste trabalho foi investigar a relação entre a atividade microbiana e o tipo de solo com a degradação e a dessorção de atrazina em Argissolo Vermelho Distrófico típico $(P V)$ e Vertissolo Ebânico Órtico chernossólico (VE), sob campo nativo, no Rio Grande do Sul. A quantidade de $15 \mathrm{~kg}$ $h a^{-1}$ de princípio ativo de atrazina foi aplicada a duas camadas de solo com diferentes teores de matéria orgânica e com umidade de $75 \%$ da capacidade de campo. A atividade microbiana foi monitorada pela evolução de $\mathrm{CO}_{2}$ e a determinação da atrazina em extratos de dessorção em metanol por cromatografia gasosa. A atividade microbiana não foi afetada pela aplicação do herbicida, sendo favorecida pelo maior teor de matéria orgânica nos solos. Aproximadamente $70 \%$ da quantidade aplicada de herbicida ficaram sorvidas ao solo, independentemente de sua classe. As taxas de degradação da atrazina lábil foram baixas e afetadas parcialmente pelo teor de carbono.

Palavras-chave: sorção, mineralização, degradação, respiração.

\section{ABSTRACT}

This study was aimed at investigating the relation between microbial activity and soil type with atrazine degradation and dessorption in samples of an Argissolo Vermelho Distrófico típico (PV) (Ultisol) and a Vertissolo Ebânico Órtico chernossólico (VE) (Mollisol) collected under native grass in the State of Rio Grande do Sul, Brazil. About $15 \mathrm{~kg} \mathrm{ha}^{-1}$ of active principle of atrazine were applied to two soil layers with different organic matter contents under $75 \%$ of humidity of the water holding capacity. The microbial activity was monitored by the $\mathrm{CO}_{2}$ evolution, and the determination of atrazine in the dessorption extracts in methanol was performed by gas chromatography. The microbial activity was not affected by the herbicide doses being stimulated by the higher organic matter content in both soils. About $70 \%$ of the applied atrazine was sorbed on both soil classes. The degradation rates of the labile atrazine were low and were partially affected by the soil carbon content.

Key words: sorption, mineralization, degradation, respiration.

\section{INTRODUÇÃO}

O herbicida atrazina (ATZ) é largamente utilizado no Rio Grande do Sul, principalmente nas culturas de milho e cana-de-açúcar. A ATZ é considerada relativamente recalcitrante no ambiente, embora a degradação microbiana seja o principal mecanismo de sua dissipação nos solos (KAUFMAN \& KEARNEY, 1970). A sorção da ATZ no solo influencia os processos de lixiviação, volatilização e mineralização (PICCOLO et al., 1998). A matéria orgânica apresentase como importante sorvente de herbicidas no ambiente. Em solos com altos teores de matéria orgânica, tem-se observado maior sorção da ATZ nos horizontes superficiais e redução na sua translocação no perfil (SHELTON et al., 1998). Elevadas correlações entre a quantidade sorvida de ATZ com o teor de matéria orgânica foram observadas em diferentes solos de região temperada (BARRIUSO et al., 1992) e em regiões tropicais (QUEIROZ \& LANÇAS, 1997).

${ }^{1}$ Curso de Pós-graduação em Ciência do Solo, Faculdade de Agronomia, Universidade Federal do Rio Grande do Sul, (UFRGS), Porto Alegre, RS, Brasil.

${ }^{2}$ Departamento de Físico-Química, Instituto de Química, UFRGS, Av. Bento Gonçalves, 9500, 91501-970, CP 15003, Porto Alegre, RS. E-mail: dpdick@iq.ufrgs.br. Autor para correspondência.

${ }^{3}$ Departamento de Solos, UFRGS. Faculdade de Agronomia, UFRGS, Porto Alegre, RS, Brasil.

${ }^{4}$ Curso de Engenharia Química, UFRGS, Porto Alegre, RS, Brasil 
Os objetivos deste trabalho foram avaliar a relação entre atividade microbiana e presença de atrazina no solo e o comportamento de dessorção do herbicida em função do tipo de solo e de seu teor de carbono.

\section{MATERIAL E MÉTODOS}

Foram coletadas amostras de solo sob campo nativo em Argissolo Vermelho Distrófico típico (PV) na Estação Experimental Agronômica da UFRGS (Eldorado do Sul, RS) e de Vertissolo Ebânico Órtico chernossólico (VE), em Bagé, RS (Tabela 1). Amostras da camada superficial $(0-20 \mathrm{~cm}$ do $\mathrm{PV}$ e $0-30 \mathrm{~cm}$ do solo VE) e de parte do horizonte B $(50-60 \mathrm{~cm}$ do PV e 30 $45 \mathrm{~cm}$ do VE) foram secadas ao ar, moídas e passadas em peneira de $4 \mathrm{~mm}$. Os tratamentos feitos em triplicata foram: $\mathrm{T}$ (sem aplicação do herbicida) e A (com aplicação do herbicida). Alíquotas de $750 \mathrm{~g}$ de solo foram acondicionadas em frascos respirométricos de $1,5 \mathrm{~L}$, e a umidade do solo foi mantida em $75 \%$ da capacidade de campo (CC).

Duas horas após o umedecimento, foi aplicado o herbicida ATZ (Atrazina Nortox 500 $\mathrm{g} \mathrm{L}^{-1}$ ) por borrifamento $(0,6 \mathrm{~mL}$ em $750 \mathrm{~g}$ solo $)$, na dose de $15 \mathrm{~kg}$ $\mathrm{ha}^{-1}$ do princípio ativo ( $10 \mathrm{x}$ a dose mínima recomendada a campo, para densidade do solo de $1 \mathrm{~g} \mathrm{~cm}^{-3} \mathrm{e}$ profundidade $0-5 \mathrm{~cm}$ ). As amostras foram incubadas em vasos envoltos em papel alumínio, o $\mathrm{CO}_{2}$ produzido foi captado em solução de $\mathrm{NaOH} 0,5 \mathrm{~mol} \mathrm{~L}^{-1}$ e quantificado por titulação com $\mathrm{HCl} 0,5 \mathrm{~mol} \mathrm{~L}^{-1}$. A respiração microbiana foi avaliada ao primeiro e aos $7,10,15,21$, 27, 35, 44, 55, 64, e 77 dias após a aplicação do herbicida.
A quantidade de $\mathrm{CO}_{2}$ liberada ( $\mathrm{mg} \mathrm{kg}^{-1}$ solo) foi calculada pela diferença entre o volume de ácido gasto na titulação da amostra e aquele gasto na prova em branco. A taxa de mineralização $\left(\mathrm{mg} \mathrm{CO} \mathrm{dia}^{-1} \mathrm{~kg}^{-1}\right.$ ) foi obtida do parâmetro angular da regressão linear baseada na relação entre a quantidade de $\mathrm{CO}_{2}$ evoluído e o tempo.

A dessorção de ATZ foi determinada ao primeiro e aos $7,15,27,35,44,55$ e 64 dias após a aplicação, em alíquotas de $50 \mathrm{~g}$ de solos retiradas em cada período e submetidas à agitação horizontal com 100mL de metanol grau HPLC (Omnisolv) por 24 horas. A suspensão foi centrifugada (3000rpm, 15min), o sobrenadante filtrado em filtro qualitativo e concentrado $(7 \mathrm{x})$ em rota-evaporador. Após a retirada da amostra do frasco respirométrico, o solo foi homogeneizado para o prosseguimento da incubação.

$\mathrm{O}$ extrato concentrado da dessorção foi novamente centrifugado e filtrado em filtro de nylon Supelco $(0,22 \mu \mathrm{m})$. A ATZ foi quantificada por cromatografia gasosa em cromatógrafo Varian 3400, com detector de ionização de chama (FID) e coluna DB 5 ( $5 \%$ dimetil-fenil-polisiloxana ( $30 \mathrm{~m} \times 0,32 \mathrm{~mm} \times 0,25 \mu \mathrm{m}$ ). As condições de análise foram determinadas previamente por ALMEIDA(2003). A curva padrão para quantificação da massa de ATZ dos extratos foi obtida com 5 soluções de ATZ de concentração conhecida no intervalo entre 0 a $30 \mathrm{mg} \mathrm{L}^{-1}$. Uma relação linear entre massa de ATZ e área do pico cromatográfico foi obtida $(\mathrm{R}=0,98)$. A quantidade mínima detectada foi de $3,6 \mathrm{mg}$ de ATZ kg-1 de solo.

Avaliando-se a quantidade de ATZ dessorvida por unidade de massa de solo em cada tempo, determinou-se a quantidade máxima de ATZ

Tabela 1 - Condições climáticas (FEPAGRO, 1990), teor de carbono total $\left(\mathrm{C}_{\mathrm{T}}\right)$, pH em água e granulometria das amostras (ALMEIDA, 2003) e proporção de ATZ máxima lábil (ATZ $\mathrm{LAB}_{\mathrm{AB}}$ ), de ATZ lábil degradada até 55 dias (ATZ $\mathrm{DGL}_{\mathrm{DL} 5}$ ), de ATZ total degradada até os 55 dias (ATZ ${ }_{\mathrm{DGT5}}$ ), de ATZ lábil remanescente até 55 dias e 64 dias (ATZ $\mathrm{AA55}_{\mathrm{e}} \mathrm{ATZ}_{\mathrm{LA64}}$ ) e ATZ fortemente sorvida (ATZ $\mathrm{Fs}_{\mathrm{F}}$ ).

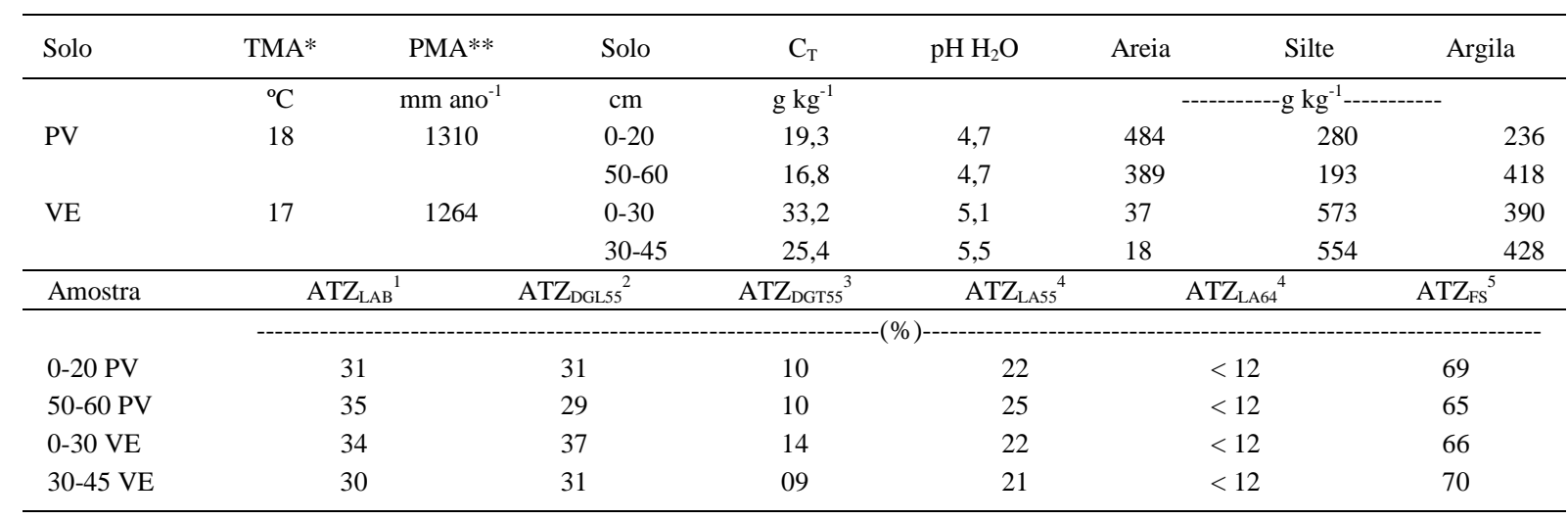

*TMA: temperatura média anual; **PMA: precipitação média anual.

(1) $\left.\mathrm{ATZ}_{\mathrm{LAB}}(\%)=\mathrm{ATZ}_{\mathrm{LA}} / \mathrm{ATZ}_{\mathrm{T}} ;(2) \mathrm{ATZ}_{\mathrm{DGL} 55}(\%)=\left(\mathrm{ATZ}_{\mathrm{LA}}-\mathrm{ATZ}_{\mathrm{DS} 55}\right) / \mathrm{ATZ}_{\mathrm{LA}} ; 3\right) \mathrm{ATZ}_{\mathrm{DGT} 55}(\%)=\left(\mathrm{ATZ} \mathrm{LA}_{\mathrm{LA}}-\mathrm{ATZ}_{\mathrm{DS} 55}\right) / \mathrm{ATZ}_{\mathrm{T}} ;(4) \mathrm{ATZ}_{\mathrm{LA} 55} \mathrm{e}$ $\mathrm{ATZ}_{\mathrm{LA} 64}(\%)=\mathrm{ATZ}_{\mathrm{DS} 55} / \mathrm{ATZ}_{\mathrm{T}}$ e ATZ $\mathrm{AS} 64_{4} / \mathrm{ATZ}_{\mathrm{T}} ;(5) \mathrm{ATZ}_{\mathrm{FS}}(\%)=\mathrm{ATZ}_{\mathrm{T}}-\mathrm{ATZ}_{\mathrm{LA}}$. 
dessorvida em cada extração e o valor considerado a quantidade máxima de ATZ lábil e, portanto, passível de degradação ( $\mathrm{ATZ}_{\mathrm{LA}}$, mg ATZ kg-1 solo). A partir de $\mathrm{ATZ}_{\mathrm{LA}}$, foram calculados: a) proporção máxima de ATZ lábil $\left(\mathrm{ATZ}_{\mathrm{LAB}}\right)$, que relaciona a quantidade máxima de $\mathrm{ATZ}_{\mathrm{LA}}$ pela quantidade total aplicada $\left(\mathrm{ATZ}_{\mathrm{T}}=30 \mathrm{mg}\right.$ $\left.\mathrm{kg}^{-1}\right)$; b) proporção de ATZ lábil que foi degradada em 55 dias $\left(\mathrm{ATZ}_{\mathrm{DGL} 55}\right)$, que relaciona a quantidade de ATZ lábil que foi degradada até os 55 dias $\left(\mathrm{ATZ}_{\mathrm{LA}}\right.$ quantidade de ATZ dessorvida aos 55 dias, $\mathrm{ATZ}_{\mathrm{DS} 55}{ }^{\mathrm{LA}}$ ) pela quantidade máxima de $\mathrm{ATZ}_{\mathrm{LA}}$; c) proporção de ATZ total degradada até 55 dias $\left(\mathrm{ATZ}_{\text {DGT55 }}\right)$, que relaciona a quantidade de ATZ lábil degradada até 55 dias $\left(\mathrm{ATZ}_{\mathrm{LA}}-\mathrm{ATZ}_{\mathrm{DS} 55}\right)$ pela quantidade total $\mathrm{ATZ}_{\mathrm{T}}$; d) proporção de ATZ lábil remanescente aos 55 dias, $\left(\mathrm{ATZ}_{\mathrm{LA55}}\right)$, ou 64 dias $\left(\mathrm{ATZ}_{\mathrm{LA64}}\right)$, que relaciona a quantidade de ATZ dessorvida aos 55 e aos 64 dias (ATZ ${ }_{\mathrm{DS} 55}$ e $\mathrm{ATZ}_{\mathrm{DS} 64}$, respectivamente) pela quantidade de ATZ ; e) proporção de ATZ fortemente sorvida ao solo $\left(\mathrm{ATZ}_{\mathrm{FS}}\right)$, que relaciona a quantidade de ATZ que não foi dessorvida $\left(\mathrm{ATZ}_{\mathrm{T}}-\mathrm{ATZ}_{\mathrm{LA}}\right)$ pela quantidade de $\mathrm{ATZ}_{\mathrm{T}}$.

Foi utilizado o delineamento completamente casualizado e as médias comparadas pelo teste de Tukey a $5 \%$ de probabilidade de erro.

\section{RESULTADOS E DISCUSSÃO}

Nos solos e nas profundidades, a mineralização de carbono (C) não foi afetada pela aplicação de ATZ, indicando que a dosagem do herbicida aplicado não afetou a população microbiana (Figura 1a). Por isso, serão usadas na discussão os valores médios.

Na camada superior do solo PV, a taxa de mineralização aos 10 dias de incubação foi de $109 \mathrm{mg}$ $\mathrm{CO}_{2} \mathrm{dia}^{-1} \mathrm{~kg}^{-1}$ solo $(\mathrm{r}=0,930)$, mais intensa que a taxa obtida entre o $10^{\circ}$ e $77^{\circ}$ dia: $59 \mathrm{mg} \mathrm{CO}_{2} \mathrm{dia}^{-1} \mathrm{~kg}^{-1}$ solo $(\mathrm{r}=0,999)$. Na camada inferior deste solo, a taxa foi relativamente constante e inferior à da camada superior: $10 \mathrm{mg} \mathrm{CO}_{2} \mathrm{dia}^{-1} \mathrm{~kg}^{-1}$ solo $(\mathrm{r}=0,940)$. No solo VE, a taxa de mineralização até o $10^{\circ}$ dia foi de 141 e $84 \mathrm{mg} \mathrm{CO}_{2}$ dia $^{-1}$ $\mathrm{kg}^{-1}$ solo a $0-30$ e $30-45 \mathrm{~cm}$, respectivamente $(\mathrm{r}=0,972 \mathrm{e}$ $\mathrm{r}=0,977$ ). Entre o $10^{\circ}$ e o $77^{\circ}$ dia, a taxa se reduziu a 78 e $47 \mathrm{mg} \mathrm{CO}_{2} \mathrm{dia}^{-1} \mathrm{~kg}^{-1}$ solo, respectivamente $(\mathrm{r} \geq 0,998)$. Esta redução com o tempo, em ambos os solos, foi atribuída ao rápido esgotamento da fração da matéria orgânica facilmente mineralizável.

Aos 77 dias de incubação, a produção de C mineralizado foi de 800 e $60 \mathrm{mg} \mathrm{kg}^{-1}$ a 0-20 e 50-60 cm no $\mathrm{PV}$, respectivamente (Figura 1a), e de 950 e $500 \mathrm{mg} \mathrm{kg}^{-1}$ a $0-30$ e $30-45 \mathrm{~cm}$ no VE, respectivamente (Figura $1 \mathrm{~b}$ ). Valores de mineralização de C de 280 e $500 \mathrm{mg} \mathrm{kg}^{-1}$ de solo foram observados em amostras de 0-20cm de um solo PV de Goiânia sob cultivo de cana-de-açúcar (ARAÚJO et al., 2001). No trabalho citado, a maior atividade metabólica dos microrganismos nas camadas superficiais do solo foi atribuída ao seu maior teor de carbono, o que, provavelmente, justifica o que foi observado no presente trabalho.

Não se verificou correlação entre teor de C no solo e quantidade de $\mathrm{C}$ mineralizado. Contudo, sabese que o decréscimo da aeração do solo em profundidade reduz a atividade microbiana devido à diminuição da oxidação de compostos orgânicos (FOLLET \& SCHIMEL, 1989). Assim, na camada superficial no PV, o baixo teor de argila propicia condição de aeração favorável à mineralização de $\mathrm{C}$, ao contrário de a $50 \mathrm{~cm}$. No solo VE, a camada subsuperficial encontra-se mais próxima da superfície do que no solo PV e é provavelmente mais aerada em situação de campo. Esta condição, aliada ao maior teor de C (Tabela 1), é favorável a uma taxa de mineralização de $\mathrm{C}$ mais intensa, comparativamente à camada subsuperficial do PV.

A dessorção de ATZ decresceu ao longo do tempo nos dois solos e profundidades (Figura 1c). No solo PV, a dessorção máxima foi na primeira extração realizada 24 horas após a aplicação. Considerando-se que este intervalo corresponde ao tempo necessário para que a sorção de ATZ no solo se complete (ALBUQUERQUE et al., 2001), esta quantidade dessorvida corresponde à quantidade máxima de ATZ lábil e passível de degradação.

No solo VE, a maior quantidade de ATZ dessorvida foi aos 15 dias, provavelmente em virtude da não ocorrência do umedecimento a $75 \%$ da CC em duas horas. Devido à presença de minerais de camada 2:1, a difusão da água no VEé mais lenta do que no PV. AATZ, devido a seu caráter hidrofóbico (Kow $=33 \mathrm{mg}$ $\mathrm{L}^{-1}$ ), deve ter migrado inicialmente para os sítios menos hidrofílicos das entrecamadas no VE, simulando uma sorção mais pronunciada logo após a aplicação. Conforme a água difundiu-se no solo, a ATZ foi deslocada das entrecamadas, tornando-se mais lábil, assim que, aos 15 dias, o umedecimento do solo se completa e a dessorção de ATZ é máxima.

A recuperação de ATZ foi baixa (30= ATZL $_{A}=35 \%$ ), sugerindo uma sorção significativa do herbicida nos solos, independentemente do teor de $\mathrm{C}$ e da mineralogia (Tabela 1). Em torno de 65 a $70 \%$ do herbicida aplicado permaneceram sorvidos ao solo (ATZFS), inviabilizando a sua degradação pela microbiota no período. A diminuição da ATZ dessorvida ao longo do tempo foi relacionada à degradação da ATZ lábil. Em todas as amostras, esta quantidade 


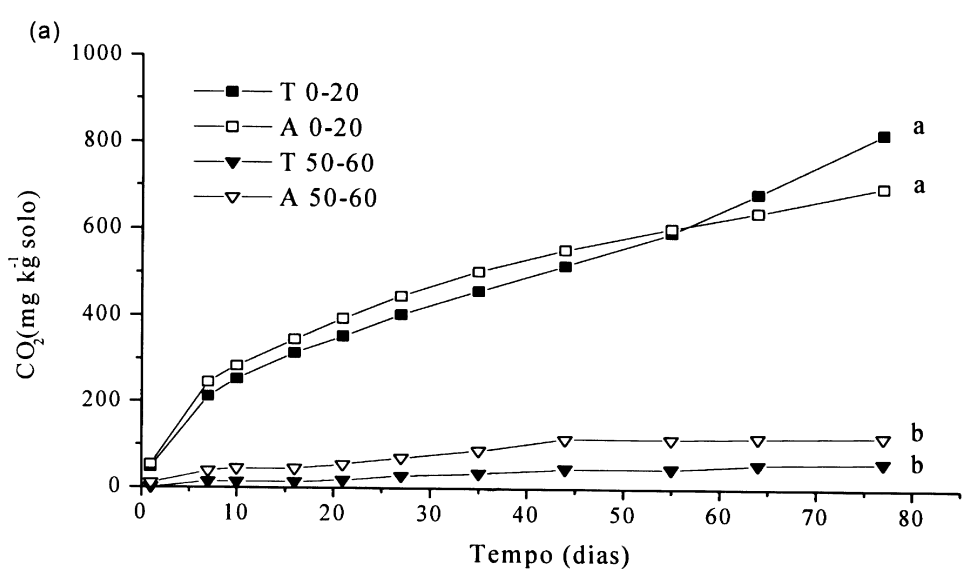

(b)

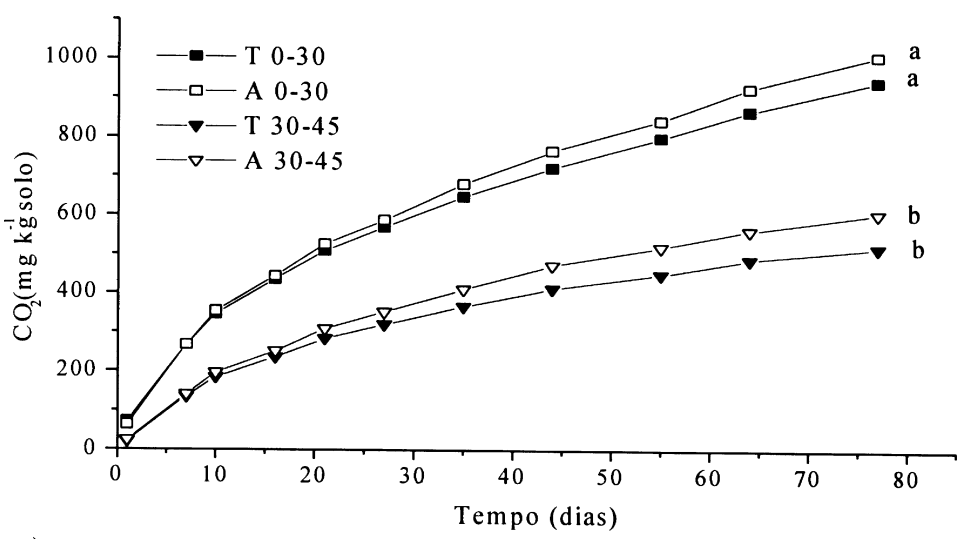

c)

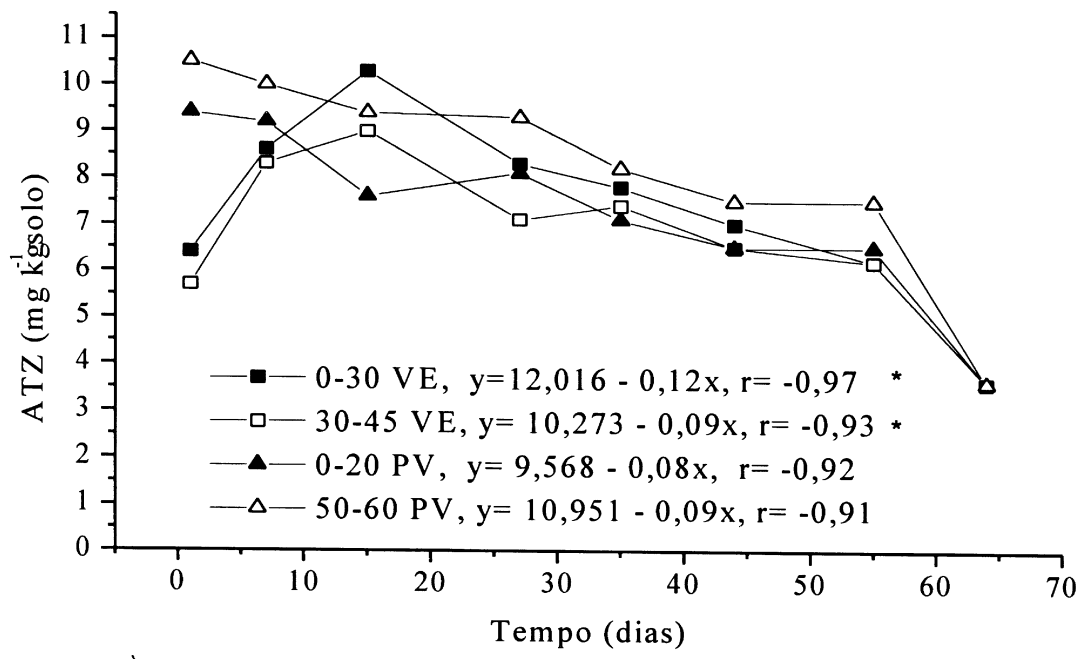

Figura 1 - Mineralização acumulada de $\mathrm{CO}_{2}$, ao longo do tempo, na ausência (T) e presença (A) do herbicida ATZ no solo PV (a), nas camadas 0-20 e 50-60 cm, e no solo VE (b), nas camadas de 0-30 e 30-45 cm, e quantidade dessorvida de ATZ em função do tempo e em camadas dos solos PV $(0-20$ e 50-60cm) e VE $(0-30$ e 30-45cm) (c). (* Dados do 1ํo 7o dia não incluídos na correlação). Médias seguidas por letras distintas diferem entre si pelo teste de Tukey a $5 \%$. 
decresceu drasticamente entre 55 e 64 dias (Figura 1c), atingindo teores abaixo do limite de detecção da técnica ao fim do experimento.

A taxa de degradação de ATZ foi inferida a partir das porções lineares das curvas (Figura 1c) e a partir do máximo de dessorção. Os valores para o solo PV foram similares nas duas camadas $\left(0,08 \leq \mathrm{mg}\right.$ ATZ $\mathrm{dia}^{-1} \mathrm{~kg}^{-1}$ solo $\left.\leq 0,09\right)$, sugerindo que o teor de carbono mais elevado e a maior atividade microbiana a 0-20cm (Figura 1a), em comparação a $50-60 \mathrm{~cm}$, não exerceram efeito pronunciado na degradação da ATZ. No solo VE, a taxa de degradação a $0-30 \mathrm{~cm}$ foi superior à verificada na camada $30-45 \mathrm{~cm}$, sendo similar ao valor de $0,14 \mathrm{mg}$ ATZ dia ${ }^{-1} \mathrm{~kg}^{-1}$ obtido em um Hapludoll, (OSTROFSKY et al., 2002). Este comportamento poderia estar relacionado com o maior teor de carbono (Tabela 1) e a maior atividade microbiana nesta camada. Porém, uma vez que não foram obtidas relações diretas entre teor de $\mathrm{C}$ e taxa de degradação de ATZ para todo o conjunto de amostras, outros fatores não abordados neste estudo podem estar afetando a decomposição do herbicida.

A proporção de ATZ lábil degradada após 55 dias de aplicação $\left(\mathrm{ATZ}_{\mathrm{DGL} 55}\right)$ variou de 29 a $31 \%$ no PV e de 31 e $37 \%$ no VE (Tabela 1). Considerando-se a quantidade total aplicada, a proporção de ATZ total degradada $\left(\mathrm{ATZ}_{\mathrm{DGT5}}\right)$ após 55 dias é de $10 \%$ no PV e de 9 e $14 \%$ a 30-45 e 0-30 cm do VE, respectivamente. A proporção de atrazina lábil ( $\mathrm{ATZ}_{\mathrm{LA55}}$ ) aos 55 dias foi semelhante nos dois solos, variando de 21 a $25 \%$ (Tabela 1). Aos 64 dias, esta forma de ATZ decresceu para $<12 \%$ em todas as amostras.

\section{CONCLUSÕES}

O decréscimo da atividade microbiana relacionou-se, em parte, com a diminuição do teor de carbono em profundidade, não sendo afetada pela aplicação de herbicida em dose 10 vezes superior à recomendada. Aproximadamente $70 \%$ de atrazina aplicada ficavam sorvidas, independentemente da classe de solo e do teor de carbono orgânico. A variação do teor de carbono explicou parcialmente a variação da taxa de degradação de atrazina.

\section{REFERÊNCIAS}

ALBUQUERQUE, M.A. et al. Mineralização e sorção de atrazina em Latossolo Roxo sob cultivo convencional e plantio direto. R Bras Ci Solo, v.25, n.1, p.179-188, 2001.

ALMEIDA, P.S.G de. Sorção de atrazina em solos de diferentes classes. 2003. 108f. Tese (Doutorado em Ciência do Solo) - Programa de Pós-graduação em Ciência do Solo, Universidade Federal do Rio Grande do Sul.

ARAÚJO, A.M.S. et al. Mineralização do C e do $\mathrm{N}$ em amostras armazenadas de solo cultivado com cana-de-açúcar ao longo de dez anos, com e sem fertilização nitrogenada. R Bras Ci Solo, v.25, n.1, p.43-53, 2001.

BARRIUSO, E. et al. Dissolved organic matter and sorption desorption of dimefuron, atrazine and carbetamide by soils. J Environ Qual, v.21, n.1, p.359-367, 1992.

FEPAGRO. Atlas agroclimático. Dados climáticos do Estado do Rio Grande do Sul. Porto Alegre, 1990. 242p.

FOLLET, R.F.; SCHIMEL, D.S. Effect of tillage practices on microbila biomass dynamics. Soil Sci Soc Am J, v.53, p.10911096, 1989.

PICCOLO, A. et al. Atrazine interactions with soil humic substances of different molecular structures. J Environ Qual, Madison, v.27, 1324-1333, 1998.

KAUFMAN, D.; KEARNEY, P.C. Microbial degradation of striazine herbicides. Residue Rev, v.32, p.235-265, 1970.

OSTROFSKY, E.B. et al. Analysis of atrazinedegrading microbial communities in soils using mostprobable-number enumeration, DNA hybridization, and inhibitors. Soil Biol Biochem, v.34, p.1449-1459, 2002 .

QUEIROZ M.E.C.; LANÇAS, F.M. HRGC. Study of sorption and desorption of atrazine, ametryn and metolachor on Brazilian Soils. J Braz Chem Soc, v.8, p.1-6, 1997.

SHELTON, D.R. et al. Effect of tillage on atrazine bioavailability. Soil Sci, v.63, n.11, p.891-896, 1998. 